\title{
Effective dynamic properties of random complex media with spherical particles
}

\author{
M. Mahbub Alam, ${ }^{1}$ Valerie J. Pinfield, ${ }^{2, a)}$ Francine Luppé, ${ }^{1}$ and Pierre Maréchal ${ }^{1}$ \\ ${ }^{1}$ Laboratoire Ondes et Milieux Complexes, Unité Mixte de Recherche 6294, Centre National de la Recherche \\ Scientifique, University of Le Havre, 76600 Le Havre, France \\ ${ }^{2}$ Chemical Engineering Department, Loughborough University, Loughborough LE11 3TU, United Kingdom
}

(Received 14 October 2018; revised 29 April 2019; accepted 28 May 2019; published online 26 June 2019)

\begin{abstract}
The effective dynamic bulk modulus and density are presented for random media consisting of particles in a viscous host fluid, using a core-shell, self-consistent effective medium model, under the large compressional wavelength assumption. These properties are relevant to acoustic or dynamic processes in nano- and micro-particle fluids including particle density determination, resonant acoustic mixing, and acoustic characterisation. Analytical expressions are obtained for the effective bulk modulus and mass density, incorporating the viscous nature of the fluid host into the core-shell model through wave mode conversion phenomena. The effective density is derived in terms of particle concentration, particle and host densities, particle size, and the acoustic and shear wavenumbers of the liquid host. The analytical expressions obtained agree with prior known results in the limit of both static and inviscid cases; the ratio of the effective bulk modulus to that of the fluid is found to be quasi-static. Numerical calculations demonstrate the dependence of the effective mass density on frequency, particle size (from nano- to micro-regime), and concentration. Herein it is demonstrated both theoretically and numerically that the viscosity, often neglected in the literature, indeed plays a significant role in the effective properties of nanofluids.
\end{abstract}

(C) 2019 Acoustical Society of America. https://doi.org/10.1121/1.5111743

[OU]

Pages: $3727-3740$

\section{INTRODUCTION}

Estimating the effective properties of complex media is of interest from both a theoretical and experimental point of view owing to their numerous applications; these include the mechanical properties of solid composite structures, the soundabsorbing properties of porous materials, and the dynamic properties of fluid-suspended particle systems. The determination of effective properties has been extensively studied for more than a century and has a vast body of literature which is reviewed briefly here. The current work is directed towards the determination of dynamic effective properties for acoustic propagation in complex fluids consisting of solid nanoparticles in a viscous liquid; previous investigations have studied mainly inviscid fluid hosts. A proper understanding of the dynamic properties of nanofluids is of great significance, for example, in the determination of particle mass or density by oscillatory methods, or in resonant acoustic mixing. Acoustic techniques are valuable for both characterisation and manipulation of nanoparticles, but the nature of interparticle interactions in a viscous fluid and their effect on acoustics is still poorly defined. Here, these effects are investigated by considering the effective bulk modulus and mass density of such a nanoparticle suspension in an acoustic field.

The effective properties of a complex medium are defined such that the behaviour of the inhomogeneous medium is equivalent to that of a homogeneous medium with those effective properties. One approach used to determine the effective

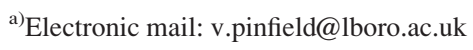

properties of random composites is to use self-consistent theories. A single inclusion is embedded within an effective homogeneous medium and self-consistency is imposed such that the physical properties (e.g., strain, stress) in the embedding medium surrounding this single inclusion are the same as those in the effective medium as a whole. While early models, such as the static self-consistent method of Hill ${ }^{1}$ and Budiansky, ${ }^{2}$ treated each inclusion as embedded directly in the infinite, homogeneous, effective medium, Christensen and $\mathrm{Lo}^{3}$ proposed a generalised self-consistent model (GSCM), originally developed by Kerner. ${ }^{4}$ The GSCM introduced a layer of the host matrix material into the equivalence model; the inclusion was treated as though embedded within a shell of host material, which was then embedded within the infinite effective medium (as illustrated in Fig. 1). Christensen ${ }^{5}$ investigated several theoretical models and concluded that the only one of these to produce acceptable results and to improve agreement with experimental data was the GSCM model; it thus received much attention and was exploited by many authors. ${ }^{6-10}$ However, these schemes were purely static, focusing on the averaging or homogenisation of physical quantities such as stress, strain, and displacement. For dynamic, wave-based problems such as acoustics, an extension of the models to include wave phenomena is necessary.

Although there are a variety of dynamic effective medium models, the most widely adopted is based on the following two hypotheses, as articulated by Kanaun et al.: ${ }^{11}$ (a) a single inclusion (coated with a shell of the host medium) behaves as if isolated and embedded in the corresponding effective medium and (b) the mean wave field in 


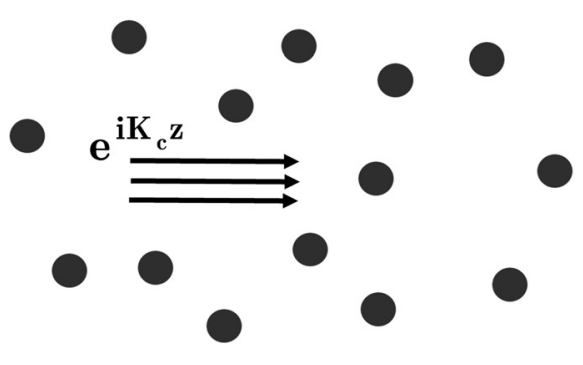

(a)

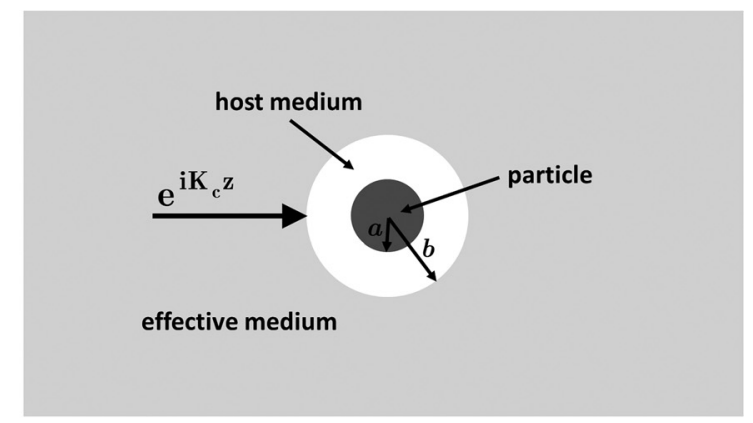

(b)

FIG. 1. Illustration of the generalised self-consistent method adopted in this work. The acoustic wave field in the complex medium (a) is equivalent to that in the effective medium surrounding a single inclusion embedded within a shell of the host medium (b). Self-consistency requires that the scattering from the core-shell system be zero.

the composite medium coincides with the wave field propagating in the homogeneous effective medium. The first hypothesis reduces the many-particle problem to a singleparticle one in the same way as the static self-consistent models, and the second one is the self-consistency condition applied to the wave field. This theory has been developed in different forms and studied by many investigators. For solid inhomogeneous systems, models include the self-consistent approaches of Kanaun et al. ${ }^{11}$ and Sabina and Willis; ${ }^{12}$ the quasi-static-limit approximations of Berryman, ${ }^{13,14}$ Kuster and Toksöz, ${ }^{15}$ Jin, ${ }^{16}$ and Mei et $a l . ;{ }^{17}$ and the dynamic approach of Gaunaurd, ${ }^{18}$ investigating solid in solid composites at low concentration (no multiple scattering) to obtain elastic and shear moduli and density, extended by $\operatorname{Kerr}^{19}$ for spherical solid inclusions, and by $\mathrm{Kim}^{20}$ to obtain frequencydependent elastic parameters. For complex fluids, i.e., liquid suspensions of particles, the effective medium method has been studied by Hemar et al. ${ }^{21}$ and McClements et al. ${ }^{22}$ to determine acoustic properties with thermal effects and Cowan et al. ${ }^{23}$ and Hipp et al. ${ }^{24}$ for acoustic systems with both thermal and shear phenomena. These workers were motivated by the characterisation of particles in suspensions using acoustic spectroscopy and the need to account for thermal and shear phenomena which were not accounted for in existing multiple scattering models.

Another approach to estimate effective dynamic properties of random distributions of particles is based upon the configurational average of the multiple scattering equations. In the following, theories using this approach are referred to as MSTs. MST was initiated by Foldy ${ }^{25}$ in 1945; he derived a dispersion relation for an acoustic wave in a system of isotropic scatterers by introducing a configurational averaging procedure. Lax $^{26}$ later extended the theory of Foldy to obtain a new dispersion relation for anisotropic scatterers and applied the quasi crystalline approximation (QCA) to determine the effective field. Waterman and Truell extended the work of Lax and Foldy to finite-sized inclusions; ${ }^{27}$ using QCA they derived an expression for the effective wavenumber in terms of the forward and backward scattering amplitude of a single particle. In order to consider higher concentration in the MSTs, Fikioris and Waterman introduced the pair correlation function in the form of "hole correction" to make sure that inclusions do not overlap during the averaging process. ${ }^{28}$
Lloyd and Berry studied vector electromagnetic fields using a Green's function approach and corrected the second-order term of Waterman-Truell formula for three dimensional particles. ${ }^{29}$ Varadan et al. formulated an MST for randomly distributed spherical $^{30}$ and cylindrical ${ }^{31}$ inclusions in an elastic matrix using QCA and a pair-correlation function to estimate effective phase velocity and coherent attenuation. More recently, Linton and Martin validated Lloyd-Berry's formula and have given a new derivation for cylindrical and spherical scatterers. $^{32,33}$ Identification of effective density and elastic properties based on multiple scattering models has also been carried out, to second order in concentration and therefore limited to low concentration. ${ }^{34-37}$

Although multiple scattering models are well-established for obtaining effective properties, they have mainly focused on ideal (inviscid) host fluids and are valid only up to a limited concentration. This is due to the assumption made in MST models that concentration is low, and effective properties are typically expressed as series in orders of the concentration, in contrast to the effective medium models where high concentrations are not constrained. However, an additional factor is the effect of thermal and shear wave modes produced by scattering of the acoustic waves by the particles; although the thickness of the thermal and viscous boundary layers are typically very small, they can affect other particles when the concentration is high and interparticle separations consequently small. These effects have been neglected in MSTs until relatively recently, when Conoir and Norris generalised the formula of Linton and Martin for cylindrical particles in elastic media, ${ }^{38}$ providing the framework to include all effective wave modes (acoustic, thermal, and shear) in the complex medium. The model was extended to three dimensions (spherical particles) at second order in concentration by Luppé et al., ${ }^{39}$ and later to higher concentrations (third order), accounting for weak pair-correlations. ${ }^{40}$ Whether in two or three dimensions, the model provides the complex wavenumbers of the coherent waves as series in the concentration, allowing thus the calculation of both the speed and the attenuation of those waves. The previously neglected multi-mode conversions have been demonstrated to be significant in nanoparticle suspensions and the new model applied to such systems $^{41}$ presents a greatly improved agreement with experimental data. ${ }^{42,43}$ Further experimental evidence of the 
need for the determination of improved effective properties is provided by a number of other workers studying acoustic propagation in liquid suspensions of particles. ${ }^{44,45}$ Moreover, determination of the effective mass density is much more complicated than in the case of an inviscid fluid host and has yet not been addressed by the model.

Since MSTs are limited to low volume concentrations, Yang and Mal combined the GSCM with the MST of Waterman and Truell in order to obtain a dynamic generalised self-consistent method (DGSCM), ${ }^{46}$ whereby they calculated the effective wavenumber of a fibre-reinforced composite (a 2D cylindrical problem) in a self-consistent manner; they showed that the effective wave speed calculated was in good agreement with experimental data. Yang ${ }^{47}$ and $\mathrm{Kim}^{48}$ used the same methodology for spherical inclusions. Other workers have also combined the two approaches, to determine effective properties at higher concentrations, but retaining the wave-nature of the problem; McClements et al. applied this approach for thermal interactions, ${ }^{22}$ and Hipp combined the two methods for a thermal-viscous-acoustic system of spherical particles although assumptions were made regarding effective properties rather than deriving them. ${ }^{24}$

This paper addresses the problem of the determination of dynamic effective properties in a shear-acoustic system of spherical particles in a viscous fluid at high concentrations, a problem requiring full account of both acoustic and shear wave modes and the impact of particle interactions due to shear boundary layer overlap. The generalised selfconsistent model described in Ref. 17, not restricted to low concentration, has been adopted and applied to a system where wave mode conversion is taken into account, under a large compressional wavelength assumption, in order to obtain analytical approximations of the effective dynamic parameters. Although use of the new GSCM model restricts the validity of the results by the assumption of long compressional wavelength, it permits a full investigation of effective properties without limit on concentration, whereas multiple scattering models are suited to low concentrations. An effective bulk modulus is obtained from the monopole mode, and an effective density from the dipole mode; for the first time, an effective density of a random medium with a viscous fluid host, that takes into account mode conversions through scattering, is presented. The assumption of large compressional wavelength applies with respect to both the particle size and the average distance between particle centres. Contrary to the case of a solid host (even viscoelastic), the shear wavelength in a viscous fluid is not the same order of magnitude as the compressional one, and the frequency dependence of the effective parameters is thus investigated by varying the ratio of that shear wavelength to the particle size over a large range, while keeping that of the compressional wave smaller than unity. While the ratio of the effective bulk modulus to that of the host is quasi-static with only limited frequencydependence, the effective density is frequency-dependent and its behaviour is investigated. In this work, the model and its principal results are presented in Sec. II, with much of the mathematical details confined to the Appendix. In Sec. III, numerical predictions of the model are presented, and their physical interpretation discussed.

\section{MODEL}

\section{A. Core-shell system for effective medium}

The system consists of a small-amplitude (linear) acoustic wave propagating through a medium consisting of identical spherical inclusions (particles) of radius $a$, randomly dispersed in an infinitely extended homogenous isotropic matrix medium with a given concentration $c$. Using the principles of the generalised self-consistent method, an equivalent problem is constructed, that of a single particle embedded in a shell of the host medium (a viscous fluid) within a homogeneous medium which has the effective properties of the system (Fig. 1). Effective properties can only be analytically derived where the acoustic wavelength is much larger than both the particle and shell sizes and indeed the average distance between centres of neighbouring particles. ${ }^{49,50}$ The radius of the shell, $b$, is fixed such that the concentration within the shell is equivalent to that in the medium as a whole, thus $b=a c^{-1 / 3}$. Thermal effects have been neglected; these are negligible in solid particle systems,${ }^{41}$ but may be significant where particles are liquid. ${ }^{22}$

The self-consistent method we use, based on the coherent potential approximation (CPA), requires that, should the outer fluid be the effective one, the scattering of the coreshell system should be minimized. ${ }^{50,51}$ Here, both acoustic (compressional or $\mathrm{P}$ modes) and shear (S) wave modes are included in all media. Thus, mode conversions are accounted for in the model. We are interested in getting explicit analytical formulas for the effective properties, so we will not use the generalized CPA as defined in Refs. 50 and 51, but instead introduce a large compressional wavelength assumption, or, more exactly, that both $\left|K_{c} b\right|$ and $\left|k_{c} b\right|$ be small compared to unity. The self-consistency condition can thus be applied independently to the Rayleigh partial wave orders, namely, the monopole and dipole modes, which are two of the dominant modes in the total scattering under the small $\left|K_{c} b\right|$ assumption. Although our work is thus limited to long acoustic wavelength, we need not apply any such assumption on the shear wavelengths, allowing us to investigate a range of shear wavelengths for incident acoustic waves and determine the frequency-dependence and/or viscosity-dependence of the effective dynamic density and bulk modulus.

Having set the monopole and dipole scattering coefficients of the core-shell system to zero, we use the same method as in Ref. 17 and work directly on the shell outerboundary conditions with no scattered waves in the outside effective medium. Considering an incident effective compressional wave that propagates in the effective medium and encounters the core-shell inclusion, we do not write any scattered wave in the effective medium, but two inwardpropagating refracted waves (one compressional and one shear with amplitudes $A_{n}^{c c}$ and $A_{n}^{c s}$, respectively) in the shell. These two refracted waves are, in turn, scattered by the core particle, each generating an outward-propagating wave of each mode in the shell and two refracted waves inside the core particle. The scattering of waves from the core particle in the host medium is characterised by scattering coefficients $T_{n}^{c c}$ and $T_{n}^{c s}$ for an incident compressional wave, and $T_{n}^{s c}$ and $T_{n}^{s s}$ for an incident shear wave. These combine with the 
incident amplitudes of the inward-propagating refracted waves in the shell $\left(A_{n}^{c c}\right.$ and $\left.A_{n}^{c s}\right)$ to define the amplitude of the outward-propagating waves in the shell.

The solution to determine the effective properties proceeds by the application of the boundary conditions at the interface of the shell and effective medium for each partial wave order independently, considering all wave modes. Rearrangement of the equations and the application of the long-wavelength requirement for the incident compressional wave leads to solutions for the effective bulk modulus and effective density. The validity of the model is not limited to a particular concentration range, but the long wavelength assumption $\left|K_{c} b\right| \ll 1$, and $\left|k_{c} b\right| \ll 1$ imposes a frequency and particle size limit which is dependent on the concentration. The practical limits imposed by this requirement are explored in the results Sec. III.

Only the main steps and the principal results are presented in the following; details of the solution can be found in the Appendix.

\section{B. Effective bulk modulus}

In order to derive an expression for the effective bulk modulus, the monopole mode (Rayleigh partial wave with $n=0$ ) with an incident compressional wave is considered. Owing to the spherical symmetry of this mode, the expansions and contractions of the particle and shell act as a source of spherical compressional waves and the motion relates closely to the difference in bulk modulus (inverse of compressibility) of the media. No shear waves exist in the monopole mode (all shear wave amplitudes are therefore zero), and the tangential displacement and stress boundary equations are identically satisfied and therefore not applied. The viscous nature of the fluid host is retained in the radial stress component and within the compressional wavenumber. The system reduces to two boundary equations which are written in Eqs. (A16) and (A17) in terms of the Lamé parameters $\lambda, \mu$, in order to relate to the bulk modulus $B=\lambda+(2 / 3) \mu$. The parameters can take complex values in order to represent a viscous material, in particular $\mu=-i \omega \eta_{s}$ for a viscous liquid. The imaginary part of the bulk modulus similarly relates to the bulk viscosity $\operatorname{Im}(B)=$ $-\omega \eta_{B}$ and its real part is the inverse of the compressibility. Primed properties are used for the particle and unprimed for the host medium in the following as well as in the Appendix. Simplification is achieved by taking only the dominant term of the spherical Bessel function for the incident wave, given the smallness of its argument, since the compressional wavelength in the effective medium is assumed to be much larger than the shell radius. The smallness of $\left|k_{c} b\right|$ assumption follows quite naturally that on $\left|K_{c} b\right|$, as both wavenumbers are usually found to be about the same order of magnitude (see, e.g., Ref. 51). Expanding the Bessel and Hankel functions for small arguments in both dimensionless compressional wavenumbers leads to an effective bulk modulus

$$
B_{e f f}=\frac{B\left(k_{c} b\right)^{3}+4 i \mu T_{0}^{c c}}{\left(k_{c} b\right)^{3}-3 i T_{0}^{c c}} .
$$

Taking the low frequency expression (small $\left|k_{c} b\right|$ ) of the scattering coefficient $T_{0}^{c c}$, given in Eq. (A1), leads to

$$
\frac{B-B_{e f f}}{3 B_{e f f}+4 \mu}=c \frac{B-B^{\prime}}{4 \mu+3 B^{\prime}} .
$$

Equation (2) is the same as that obtained by Kuster and Toksöz for solid-in-solid systems. ${ }^{15}$

For an elastic solid host medium, Eq. (2) shows that the effective bulk modulus $B_{\text {eff }}$ does not vary with frequency. Similarly, considering an inviscid liquid as the host medium, $\mu \rightarrow 0, B \rightarrow \lambda$, and the effective bulk modulus is again independent of frequency and reduces to the harmonic mean of the component phases weighted by concentration, as found by Mei and Aristegui for cylindrical ${ }^{17}$ and spherical ${ }^{37}$ scatterers, respectively,

$$
\frac{1}{B_{e f f}}=\frac{(1-c)}{B}+\frac{c}{B^{\prime}} .
$$

This bulk modulus expression appears in the Wood's formula for sound velocity. ${ }^{52}$

For a viscous liquid host medium with elastic solid particles, taking the complex shear modulus $\mu=-i \omega \eta_{s}$ results in a scattering coefficient such that $T_{0}^{c c} /\left(k_{c} b\right)^{3}$ is again quasi-frequency-independent (consistent with that presented by Allegra and Hawley ${ }^{53}$ ), and Eq. (1) shows in that case that the ratio of the effective bulk modulus to that of the host is also quasi-static.

\section{Effective dynamic mass density}

The effective dynamic mass density has been investigated extensively over a number of years, with many researchers finding its value to differ appreciably from the volume average mass density ${ }^{14-17,28,35-37,54-57}$ even in the zero frequency limit, unless studying solid-in-solid composites. The difference in inertia between the particles and the background medium causes the particles to move relative to the host matrix; due to this periodic oscillation, the particles mostly radiate in the forward and backward directions, known as dipole radiation. The difference in the relative motion caused by the restoring force in the elastic or viscous fluid host medium implies that the effective dynamic mass density is in general a complex quantity.

The effective dynamic mass density can be determined from the dipole mode $n=1$ by considering a compressional wave mode incident on the single core-shell system embedded within the effective medium. Both compressional and shear wave modes exist in all regions for the dipole partial wave order, except the scattered ones in the effective outer medium, where their amplitudes are set to zero. Four boundary conditions are applied at the shell surface [Eqs. (A18)-(A21) of the Appendix]; in this case, these are expressed in terms of wavenumbers and densities, rather than the Lamé parameters as were used for the monopole mode. Again, simplification is achieved by applying the long compressional wavelength condition. Frequency dependence appears through the variation of the shear wavelength relative to the particle size through the parameter $k_{s} a$ which is not restricted to small values. Four sets of results are derived here: 
(1) by rearrangement of the boundary equations and applying an assumption on the amplitudes of the waves in the shell (an assumption validated in Sec. III) to derive a frequency-dependent effective density;

(2) by analytical solution of the boundary equations using series expansions for all functions of compressional wave modes and retaining the leading order term for the scattering coefficients - this proves the assumption made in (1) and results in an effective density expression which is the leading order in $k_{c} b$;

(3) by expanding the expression for the effective density obtained in (2) using series expansions in the shear wavenumbers in the liquid host, to obtain the low frequency expression of the effective density when $\left|k_{s} a\right|$ is small;

(4) by using the large argument asymptotic expansion of the Bessel and Hankel functions related to shear waves in the viscous liquid host in order to recover Ament's formula for the effective mass density in the case of an inviscid fluid, corresponding to infinitely large $\left|k_{s} a\right|$.

Each of these is presented in the following, with fuller details in the Appendix.

\section{General expression for the effective density}

By combining the boundary equations Eqs. (A18)-(A21) as explained in the Appendix, the effective density is obtained in terms of the coefficients of the scattered waves in the shell as

$$
\frac{\rho_{\text {eff }}}{\rho}=\frac{E}{F}
$$

where

$$
\begin{aligned}
E= & D\left[j_{1}\left(k_{c} b\right)+T_{1}^{c c} h_{1}\left(k_{c} b\right)+2 T_{1}^{c s} h_{1}\left(k_{s} b\right)\right] \\
& +\left[2 j_{1}\left(k_{s} b\right)+T_{1}^{s c} h_{1}\left(k_{c} b\right)+2 T_{1}^{s s} h_{1}\left(k_{s} b\right)\right], \\
F= & D\left\{j_{1}\left(k_{c} b\right)+T_{1}^{c c} h_{1}\left(k_{c} b\right)\right. \\
& \left.+T_{1}^{c s}\left[k_{s} b h_{1}^{\prime}\left(k_{s} b\right)+h_{1}\left(k_{s} b\right)\right]\right\} \\
& +\left[k_{s} b j_{1}^{\prime}\left(k_{s} b\right)+j_{1}\left(k_{s} b\right)\right] \\
& +T_{1}^{s c} h_{1}\left(k_{c} b\right)+T_{1}^{s s}\left[k_{s} b h_{1}^{\prime}\left(k_{s} b\right)+h_{1}\left(k_{s} b\right)\right] .
\end{aligned}
$$

The ratio of amplitudes

$$
D=\frac{A_{1}^{c c}}{A_{1}^{c s}}
$$

can be expressed, under assumptions based on the large compressional wavelength conditions as detailed in the Appendix and validated in Sec. III, as

$$
D=\frac{k_{s} b j_{1}^{\prime}\left(k_{s} b\right)-j_{1}\left(k_{s} b\right)-T_{1}^{s c}\left[k_{c} b h_{1}^{\prime}\left(k_{c} b\right)-h_{1}\left(k_{c} b\right)\right]+T_{1}^{s s}\left[k_{s} b h_{1}^{\prime}\left(k_{s} b\right)-h_{1}\left(k_{s} b\right)\right]}{k_{c} b j_{1}^{\prime}\left(k_{c} b\right)-j_{1}\left(k_{c} b\right)+T_{1}^{c c}\left[k_{c} b h_{1}^{\prime}\left(k_{c} b\right)-h_{1}\left(k_{c} b\right)\right]-T_{1}^{c s}\left[k_{s} b h_{1}^{\prime}\left(k_{s} b\right)-h_{1}\left(k_{s} b\right)\right]} .
$$

Equations (4)-(7) have been obtained with no restriction as to the magnitude of the dimensionless shear wavenumber $k_{s} b$.

\section{Leading order in compressional wavenumber}

In order to validate the assumptions made in order to get Eq. (7), the boundary equations were solved analytically using MAPLE software, and expressed as series solutions in the compressional wavenumber, keeping only the leading order terms of the scattering coefficients in $k_{c} a$ as in Ref. 41 and given in Eqs. (A2)-(A7), leading to

$$
\begin{aligned}
\frac{\rho_{e f f}}{\rho} & =1+9 i \delta / \chi, \\
\delta= & \left(-T_{1}^{c c} T_{1}^{s s}+T_{1}^{s c} T_{1}^{c s}\right) \beta_{h b}-T_{1}^{c c} \beta_{j b}, \\
\chi= & \left(k_{c} b\right)^{3} \beta_{j b}-3 i\left(k_{c} b\right) T_{1}^{s c}+\left(k_{c} b\right)^{3} T_{1}^{s s} \beta_{h b} \\
& -6 i\left(-T_{1}^{c c} T_{1}^{s s}+T_{1}^{s c} T_{1}^{c s}\right) \gamma_{h b}+6 i T_{1}^{c c} \gamma_{j b}-6 i \frac{\left(k_{c} b\right)^{2}}{\left(k_{s} b\right)} T_{1}^{c s},
\end{aligned}
$$

with

$$
\begin{aligned}
& \beta_{h b}=k_{s} b h_{1}^{\prime}\left(k_{s} b\right)-h_{1}\left(k_{s} b\right), \\
& \beta_{j b}=k_{s} b j_{1}^{\prime}\left(k_{s} b\right)-j_{1}\left(k_{s} b\right),
\end{aligned}
$$

$$
\begin{aligned}
& \gamma_{h b}=k_{s} b h_{1}^{\prime}\left(k_{s} b\right)+2 h_{1}\left(k_{s} b\right), \\
& \gamma_{j b}=k_{s} b j_{1}^{\prime}\left(k_{s} b\right)+2 j_{1}\left(k_{s} b\right) .
\end{aligned}
$$

Two solutions have now been presented for the dynamic effective density, accounting for mode conversions between compressional and shear wave modes: the general Eq. (4), and its leading order in $k_{c} b$, Eq. (8). Both are limited to small compressional wavenumber $k_{c} b$, but no assumption has yet been made on the magnitude of the dimensionless shear wavenumber $k_{s} b$, which, in a fluid host medium, can range from small to large values within the long compressional wavelength region. The frequency-dependent behaviour of the effective density will be explored in Sec. III, but consideration is first given explicitly to the low frequency region where the shear wavelength is large compared with the particle size, as well as the converse case of an inviscid host fluid where the shear wavelength tends to zero.

\section{Low frequency expansion in shear wavenumber}

Starting from Eq. (8) all Bessel and Hankel functions with argument $k_{s} b$ are expanded as series in the dimensionless shear wavenumber which is now taken to be small. Further details are given in the Appendix. 
With these low-frequency approximations, one can show that the effective mass density reduces to the following expression:

$$
\begin{aligned}
\frac{\rho_{\text {effL }}}{\rho}\left(k_{s} a\right)= & (1-c)+c \hat{\rho}+J(c)\left(k_{s} a\right)^{2} \\
& +K(c)\left(k_{s} a\right)^{4}+O\left(\left(k_{s} a\right)^{6}\right),
\end{aligned}
$$

where

$$
\begin{aligned}
J(c)= & -\frac{c}{18} \frac{(\hat{\rho}-1)^{2}}{\left(c^{5 / 3}-1\right)^{2}}\left[4 c^{11 / 3}-9 c^{10 / 3}\right. \\
& \left.+10 c^{8 / 3}+13 c^{5 / 3}-13 c^{2}-10 c+9 c^{1 / 3}-4\right]
\end{aligned}
$$

and

$$
K(c)=\frac{1}{2268} \frac{(\hat{\rho}-1)^{2}}{\left(c^{5 / 3}-1\right)^{3}}\left[\begin{array}{l}
-81 c^{2 / 3}+(-112 \hat{\rho}+448) c+(504 \hat{\rho}-909) c^{4 / 3} \\
+(-567 \hat{\rho}+567) c^{5 / 3}+(-560 \hat{\rho}+605) c^{2} \\
+(1260 \hat{\rho}-612) c^{7 / 3}+(616 \hat{\rho}-1519) c^{8 / 3} \\
+(-2562 \hat{\rho}+2922) c^{3}+(1071 \hat{\rho}-1071) c^{10 / 3} \\
+(1820 \hat{\rho}-1460) c^{11 / 3}+(-1820 \hat{\rho}+917) c^{4} \\
+(-1071 \hat{\rho}+1719) c^{13 / 3}+(2562 \hat{\rho}-2517) c^{14 / 3} \\
+(-616 \hat{\rho}+616) c^{5}+(-1260 \hat{\rho}+855) c^{16 / 3} \\
+(560 \hat{\rho}-224) c^{17 / 3}+(567 \hat{\rho}-648) c^{6} \\
+(-504 \hat{\rho}+504) c^{19 / 3}+(112 \hat{\rho}-112) c^{20 / 3}
\end{array}\right] .
$$

As expected, ${ }^{14,17}$ Eq. (11) provides the static limit of the effective density as the volume averaged density,

$$
\lim _{\substack{k_{c} b \rightarrow 0 \\ k_{s} b \rightarrow 0}} \frac{\rho_{e f f}}{\rho}=(1-c)+c \hat{\rho},
$$

which is indeed the addition law of densities. Since $\left(k_{s} a\right)^{2}$ is purely imaginary for a viscous liquid, the leading-order frequency dependence in Eq. (11) provides only the imaginary part of the effective density; the real part varies only as $\left(k_{s} a\right)^{4}$. The concentration dependence, Eqs. (12), (13), is complicated, and features terms in one-third powers of the concentration, which is typical for such core-shell self-consistent models. This is in contrast to the multiple scattering models that usually provide effective properties at low concentration and are often expressed as series in integer orders of concentration.

Having determined the effective density in the longshear-wavelength limit (small $k_{s} a$ ), the case of an inviscid host fluid, for which $k_{s} a$ tends to infinity, is now examined.

\section{The limit of an inviscid liquid host}

Starting again from Eq. (8), the effective density is now considered in the limit as $\left|k_{s} a\right|$ tends to infinity, i.e., equivalent to the liquid host tending to an inviscid fluid. By using the asymptotic expansions of the Bessel and Hankel functions for large $\left|k_{s} b\right|$, Eq. (8) provides (see Appendix for details)

$$
\frac{\rho_{e f f}}{\rho} \sim 1-9 i c \frac{T_{1}^{c c}}{\left(k_{c} a\right)^{3}+6 i c T_{1}^{c c}} .
$$

Taking the limiting form of the compressional mode coefficient $T_{1}^{c c}$ for large $\left|k_{s} a\right|$ [Eq. (A10)], Eq. (15) results in

$$
\frac{\rho_{e f f}}{\rho}=\frac{\left(\rho+2 \rho^{\prime}\right)+c\left(\rho^{\prime}-\rho\right)}{\left(\rho+2 \rho^{\prime}\right)-2 c\left(\rho^{\prime}-\rho\right)},
$$

which is identical to Ament's formula (for spherical particles) for an inviscid fluid host. ${ }^{54}$ The same results for effective density as Eqs. (15) and (16) are obtained by applying the self-consistent scheme directly in the case of an inviscid fluid host as shown in the Appendix. Hence our expression for effective density tends to the expected static limit for an inviscid fluid host when $\left|k_{s} a\right|$ tends to infinity (still requiring $\left.\left|k_{c} a\right| \ll 1\right)$.

\section{RESULTS OF NUMERICAL CALCULATIONS}

Numerical calculations were carried out in MATLAB (v2017a) for silica particles in water at $25^{\circ} \mathrm{C}$. The material properties of silica and water used are shown in Table I. Only the effective density is investigated numerically since

TABLE I. Physical properties of silica and water at $25^{\circ} \mathrm{C}$ (298.15 K). Data for water are from Ref. 58 and for silica from Ref. 59 with a modified silica density, based on experimental measurement (Ref. 42).

\begin{tabular}{lcc}
\hline \hline & Water & Silica \\
\hline Sound velocity $\left(\mathrm{m} \mathrm{s}^{-1}\right)$ & 1497.0 & 5968.0 \\
Density $\left(\mathrm{kg} \mathrm{m}^{-3}\right)$ & 997.0 & 2100.0 \\
Shear viscosity $(\mathrm{Pa} \mathrm{s})$ & 0.000891 & 0.0 \\
Shear modulus $(\mathrm{GPa})$ & 0.0 & 30.9 \\
Attenuation coefficient $\left(\mathrm{Np} \mathrm{m}^{-1} \mathrm{MHz}^{-2}\right)$ & 0.023 & $2.6 \times 10^{-10}$ \\
\hline \hline
\end{tabular}




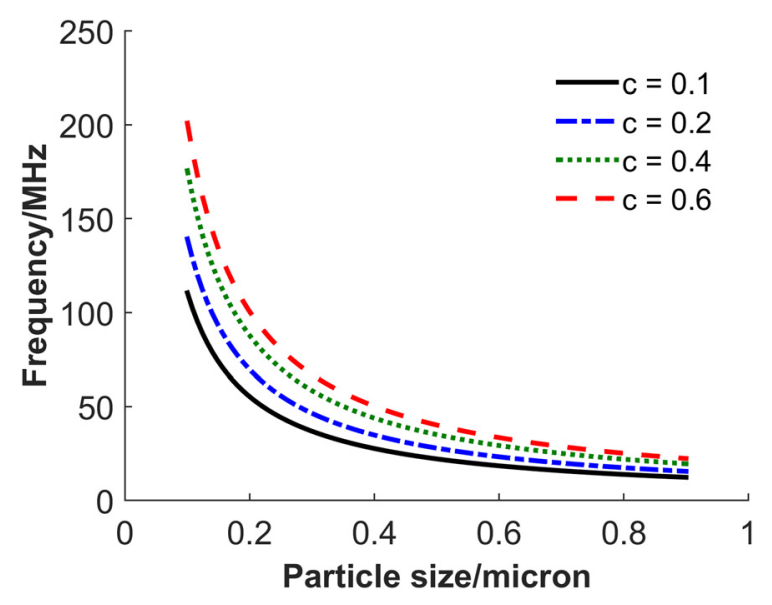

FIG. 2. (Color online) Range of validity of the model in terms of particle size and frequency at different concentrations, satisfying the condition $\left|k_{c} b\right| \leq 0.1$. The condition is satisfied below the curves.

the ratio of the effective bulk modulus to that of the host is almost frequency-independent at fixed concentration. The three expressions obtained for the effective density, Eq. (4), Eq. (8), and the low frequency expansion for small $\left|k_{s} a\right|, \mathrm{Eq}$. (11), are compared. Both frequency and concentration dependence are investigated. ${ }^{58,59}$

The effective mass density, derived under the large compressional wavelength assumption, depends on concentration and on the ratios, to the particles size, of both the compressional and the shear wavelengths in the host. In order to demonstrate the effect of this condition on the range of validity of the model in terms of particle size, frequency, and concentration, we write the condition as $\left|k_{c} b\right|<\left|k_{c} b\right|_{\max }$ with $\left|k_{c} b\right|_{\max }=0.1$. Since the imaginary part of $k_{c}$ is small, compared to its real part, we thus obtain a validity condition

$$
\text { af }<\left|k_{c} b\right|_{\max } \frac{v}{2 \pi} c^{1 / 3}
$$

The ranges of particle size and frequency over which the model is therefore valid are shown in Fig. 2 indicating a useful and workable range of validity, which improves at higher concentration (as the shell becomes smaller with fixed particle size).

While the shear wavelength in an elastic particle is the same order of magnitude as the compressional wavelength, this is not the case in a viscous fluid, and we want to investigate a large range of $k_{s} a$ values (as encountered in experimental conditions). However, investigating small shear wavelengths and large compressional wavelength is possible only for sufficiently large particles. Applying the validity condition above implies that for a fixed particle size $a_{0}$ the maximum value that $\Re e\left(k_{s} a\right)$ can reach is approximately given by

$$
\left(\Re e\left(k_{s} a\right)\right)_{\max }^{2}=\frac{1}{2}\left|k_{c} b\right|_{\max } \frac{\left|k_{s}^{2}\right|}{\left|k_{c}\right|} c^{1 / 3} a_{0} .
$$

Conversely, setting both $\left|k_{c} b\right|_{\max }$ and the maximum value of $\Re e\left(k_{s} a\right)$ one is interested with, Eq. (18) provides the minimum value $a_{0}$ that the particle radius can take.

\section{A. Dependence on frequency at different particle sizes}

First the frequency dependence of the effective density at fixed concentration is investigated. Setting $\left(\Re e\left(k_{s} a\right)\right)_{\max }$ to $100,\left|k_{c} b\right|_{\max }$ to 0.1 , and concentration $c$ to 0.4 , Eq. (18) provides a minimum value for the particle radius approximately equal to $160 \mu \mathrm{m}$. Figure 3 shows the effective mass density variation with the real part of $k_{s} a$, up to 100 , for smaller values of radius, ranging from $50 \mathrm{~nm}$ to $100 \mu \mathrm{m}$ along with the maximum values that $\Re e\left(k_{s} a\right)$ can reach in each case, according to Eq. (18). While the curves of the effective mass density depend only on the reduced wavenumbers and, thus, not on the particular elastic and viscous fluid media we are dealing with, the maximum values of $\Re e\left(k_{s} a\right)$ that can be reached for different values of $a_{0}$ depend on those, through the $\left|k_{s}^{2}\right| /\left|k_{c}\right|$ ratio in Eq. (18).

Equations (4) and (8) agree over the full range, until the limit of $\left|k_{c} b\right| \leq 0.1$ is reached (not shown on the plot). Figure 3 shows that the low frequency expansion, Eq. (11), provides quite accurate results even up to values of $\operatorname{Re}\left(k_{s} a\right)$

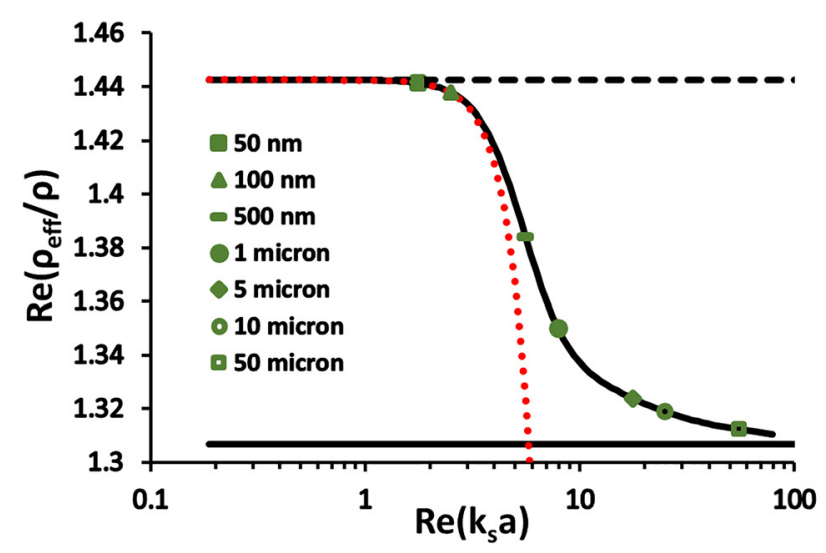

(a)

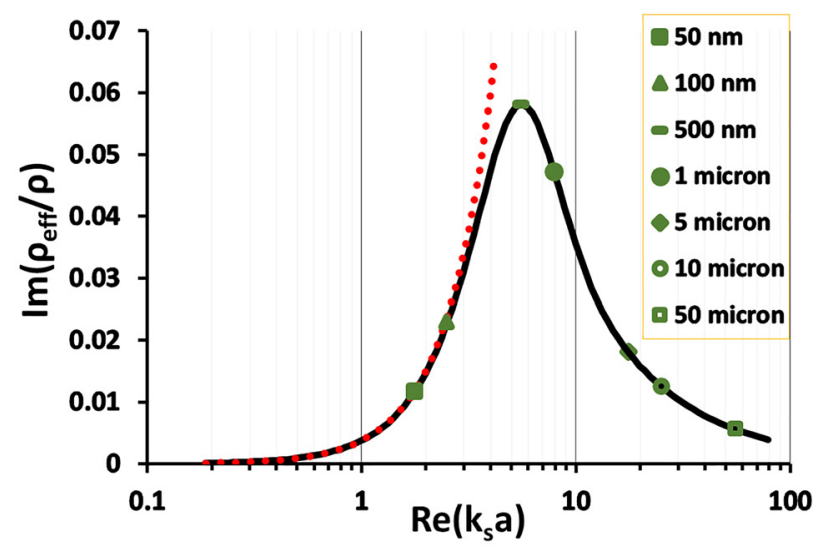

(b)

FIG. 3. (Color online) The real (a) and imaginary (b) parts of the normalised effective density for silica particles in water at $40 \% \mathrm{v} / \mathrm{v}$ concentration, as a function of the dimensionless shear wavenumber $k_{s} a$. The solid curve shows the result of Eq. (8); the dotted line shows the low frequency expansion in Eq. (11). The dashed line shows the static limit [from Eq. (14)], the solid (constant) black line Ament's static limit, Eq. (16), for the inviscid fluid. The curves were calculated using a particle radius of $100 \mu \mathrm{m}$; the symbols show the upper limit of $k_{s} a$ for particles of different radii satisfying the condition $\left|k_{c} b\right| \leq 0.1$. 


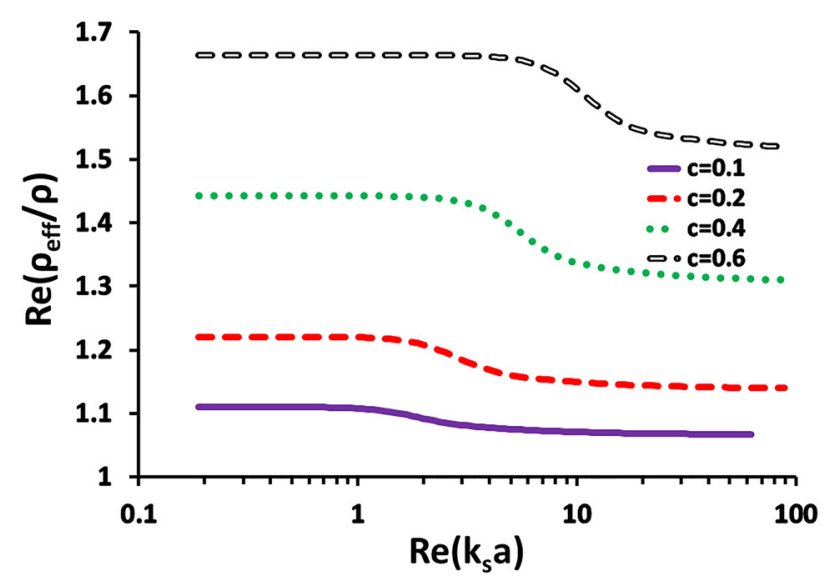

(a)

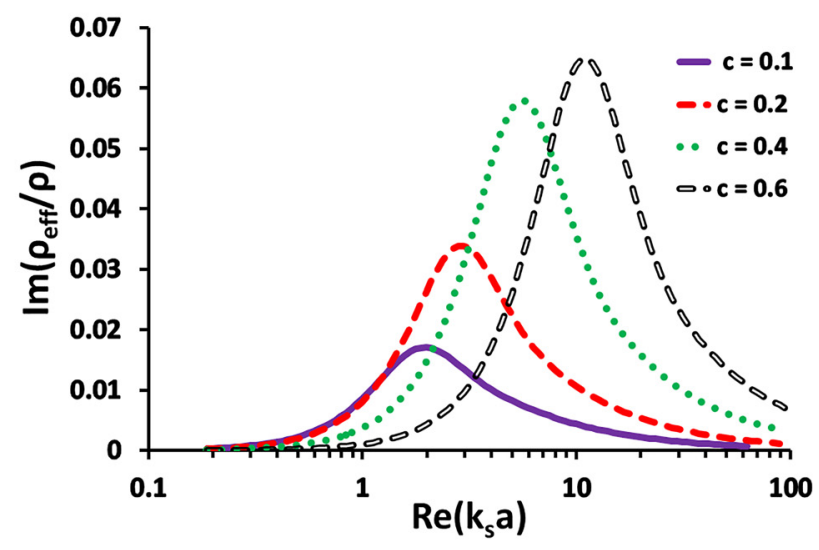

(b)

FIG. 4. (Color online) The real (a) and imaginary (b) parts of the normalised effective density given by Eq. (8) for silica particles in water at different concentrations, as a function of the dimensionless shear wavenumber $k_{s} a$. The curves were calculated using a particle radius of $100 \mu$ m; smaller particles have an upper limit of validity in $\operatorname{Re}\left(k_{s} a\right)$ in order to satisfy the condition $\left|k_{c} b\right| \leq 0.1$, as shown in Fig. 3 .

slightly larger than unity. The effective density is seen to tend to the volume averaged mass density [Eq. (14)] at small $\left|k_{s} a\right|$, and to that of Ament's formula for the inviscid fluid host, Eq. (16), at large $\left|k_{s} a\right|$. While the smaller the particles the smaller the range of $k_{s} a$, where the results are valid, one can see the evolution of the effective density from its static value to the dynamic range with the increase of $k_{s} a$, even for nanoparticles such that $a \leq 100 \mathrm{~nm}$.

The intermediate parts of the curves (Fig. 3) show a peak in the imaginary part of the effective density corresponding to an inflection point in the real part, for $\Re e\left(k_{s} a\right)$ slightly larger than unity. Single particle scattering theory shows that the scattering coefficients involving a shear wave undergo a resonance-like behavior against frequency at $\operatorname{Re}\left(k_{s} a\right) \simeq 1 .^{53}$ The effect of such a behavior has been observed on the compressional coherent wave properties predicted both by multiple scattering models that consider only multiple scattering of the acoustic mode, such as Lloyd and Berry's model, ${ }^{29}$ and by the multi-mode scattering model of Luppé et al. $^{39}$ (see discussions in Ref. 41). The same kind of effect is seen here on the effective mass density.

\section{B. Dependence on frequency at different concentrations}

Having investigated the frequency dependence of the effective mass density at fixed concentration, Fig. 4 now shows how this frequency dependence is influenced by a change in the particle concentration. Two observations can be made as the concentration increases (i) the change of magnitude in the real and imaginary parts of the mass density occurs at higher values of $\operatorname{Re}\left(k_{s} a\right)$ (i.e., higher frequency at fixed particle size) and (ii) the amplitude of the peak in the imaginary part increases, as well as the difference between static and inviscid limits on the real part.

Recent numerical investigation by Pinfield's group ${ }^{41}$ and experimental validation (on acoustic attenuation) $)^{42,43}$ have demonstrated that the resonance peak observed in the attenuation against frequency curve for the coherent compressional wave also shifts to higher $\operatorname{Re}\left(k_{s} a\right)$ as the concentration increases. This is consistent with the findings here on the effect of concentration on the effective density, which influences the effective compressional wavenumber. Physically, the effect relates to the effective stiffening of the medium due to the increase in viscous drag caused by the presence of a higher density of particles in the medium. This increased drag has been observed in a concentrated particle suspension and the corresponding viscosity increase, modelled by many workers including Happel and others (see review in Ref. 59). The shift to higher $\operatorname{Re}\left(k_{s} a\right)$ of the resonance peak of both the properties of the coherent compressional wave and the effective density as the concentration increases indicates that the resonance frequency is driven by the wavelength to particle size ratio of the shear wave in the effective medium rather than that in the host medium.

\section{Dependence on concentration at fixed $\boldsymbol{k}_{s} a$}

In order to explore the concentration dependence further, different regions around the resonance condition are investigated, selecting fixed values of $\operatorname{Re}\left(k_{s} a\right)$ either side of the resonance peak, near the static limit, near the inviscid limit and near the resonance condition. Since the long compressional wavelength condition must be satisfied $\left(\left|k_{c} b\right| \leq 0.1\right)$, and the shell radius becomes larger as the concentration decreases, the condition becomes more stringent on particle size and frequency at low concentrations. Thus, particle sizes and frequencies have been selected [for a given value of $\left.\operatorname{Re}\left(k_{s} a\right)\right]$ such that the $\left|k_{c} b\right| \leq 0.1$ condition is

TABLE II. System parameters for investigation of concentrationdependence of the effective density at fixed $\operatorname{Re}\left(k_{s} a\right)$.

\begin{tabular}{lccc}
\hline \hline $\operatorname{Re}\left(k_{s} a\right)$ & $\begin{array}{c}\text { Particle } \\
\text { radius }(\mathrm{nm})\end{array}$ & $\begin{array}{c}\text { Frequency } \\
(\mathrm{MHz})\end{array}$ & $\begin{array}{c}\text { Minimum } \\
\text { concentration }(\% \mathrm{v} / \mathrm{v})\end{array}$ \\
\hline 1 & 50 & 113.9 & 1.4 \\
2 & 150 & 50.6 & 3.2 \\
5 & 800 & 11.1 & 5.2 \\
20 & 13000 & 0.67 & 5.0 \\
\hline \hline
\end{tabular}




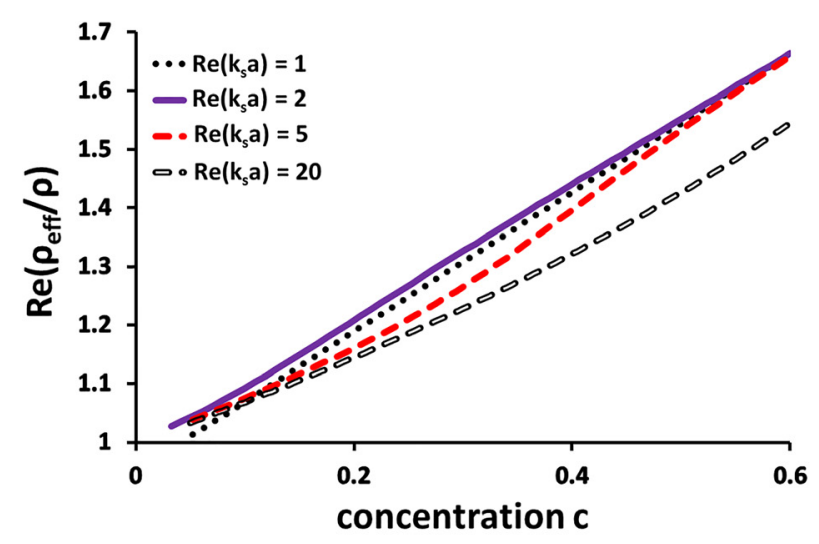

(a)

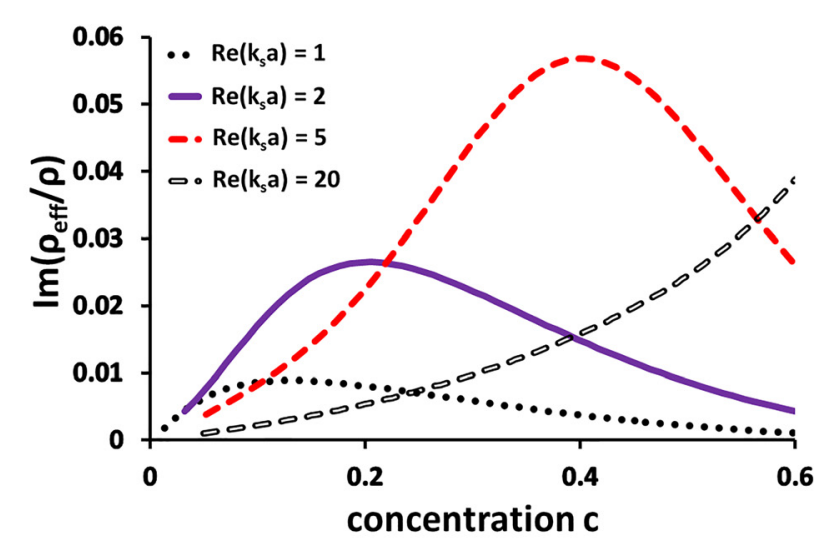

(b)

FIG. 5. (Color online) The real (a) and imaginary (b) parts of the normalised effective density from Eq. (8) for silica particles in water as a function of concentration, at fixed values of the dimensionless shear wavenumber $\operatorname{Re}\left(k_{s} a\right)$.

satisfied for concentrations greater than $c_{\min }=0.05(5 \% \mathrm{v} / \mathrm{v})$. The long compressional wavelength condition is satisfied by particles obeying Eq. (17) with $c=c_{m i n}$.

A particle radius is selected to satisfy this condition for each value of $\operatorname{Re}\left(k_{s} a\right)$; the frequency is then deduced from $a$ and $\operatorname{Re}\left(k_{s} a\right)$. Thus, the data presented all satisfy the long compressional wavelength condition $\left|k_{c} b\right| \leq 0.1$ for concentrations above $5 \% \mathrm{v} / \mathrm{v}$ and some for smaller concentrations. Table II shows the set of values of particle radius and frequency that were used for the calculations shown in Fig. 5, along with the corresponding minimum concentration satisfying the $\left|k_{c} b\right| \leq 0.1$ condition.

The real and imaginary parts of the effective density are shown in Fig. 5 as a function of concentration for several values of the dimensionless shear wavenumber. At small and intermediate values of $\operatorname{Re}\left(k_{s} a\right)$, the real part of the effective density is almost linear, and is determined largely by the linear variation of the static limit of Eq. (14). The rather weak frequency-dependence is manifested only at $\operatorname{larger} \operatorname{Re}\left(k_{s} a\right)$, where some curvature is seen and the real part of the effective density is observed to deviate significantly from the static value. The imaginary part of the effective density

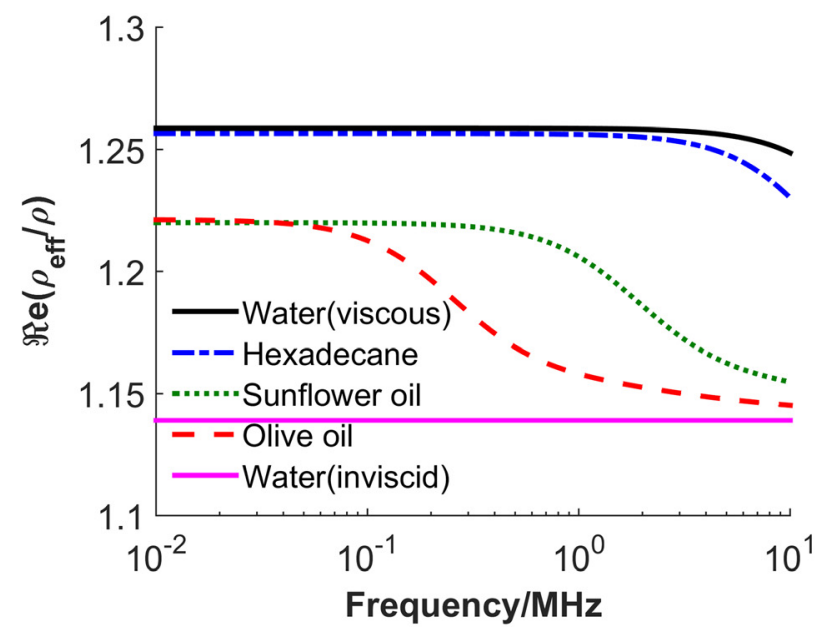

(a) shows a peak in all but the highest $\operatorname{Re}\left(k_{s} a\right)$ curves. As already observed in Fig. 4(b), the magnitude of the peaks increases with concentration. The peaks occur for couples of $\left(\operatorname{Re}\left(k_{s} a\right), c\right)$ values roughly the same as in Fig. 4(b). For example, at $\operatorname{Re}\left(k_{s} a\right)=5$, the peak in the imaginary part of the effective density curve with concentration occurs at $c=0.4$ in Fig. 5(b). At this concentration, it occurred in the curve of Fig. 3(b) against $\operatorname{Re}\left(k_{s} a\right)$ at $\operatorname{Re}\left(k_{s} a\right)=5.6$. At the largest particle size, all physically realistic concentration conditions occur to the inviscid limit side of the resonance peak in $\operatorname{Re}\left(k_{s} a\right)$ and the concentration dependence is therefore monotonically increasing with concentration.

\section{Dependence on frequency with different viscosities}

In order to show how the effective density may be affected by changes in viscosity of the fluid host medium, we show its dependence as a function of frequency in Fig. 6 for a particle size of $3 \mu \mathrm{m}$, concentration $25 \%$, and with different host fluids (properties shown in Table III). The density contrast between particles and fluid is different in each case, hence the static and inviscid limits are also different. As

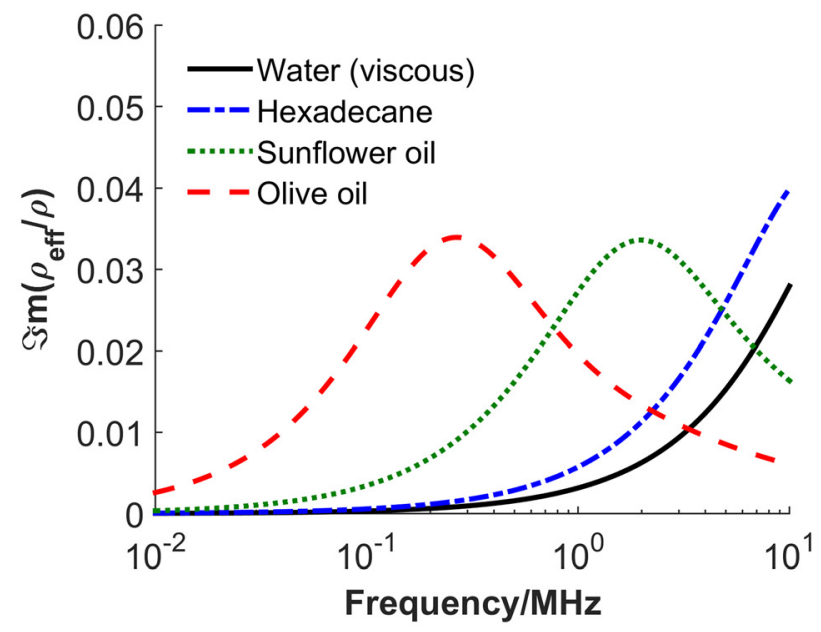

(b)

FIG. 6. (Color online) The real (a) and imaginary (b) parts of the normalised effective density as a function of frequency from Eq. (8) for silica particles in various viscous fluids, for a particle radius of $3 \mu \mathrm{m}$ and $25 \%$ concentration. 
TABLE III. Host fluid properties for investigation of viscosity-dependence of the effective density.

\begin{tabular}{|c|c|c|c|c|}
\hline Host fluid & Water (Ref. 59) & Hexadecane (Ref. 59) & Sunflower oil (Ref. 58) & Olive oil (Ref. 63) \\
\hline Sound velocity $\left(\mathrm{m} \mathrm{s}^{-1}\right)$ & 1497 & 1299 & 1470 & 1464 \\
\hline Density $\left(\mathrm{kg} \mathrm{m}^{-3}\right)$ & 997 & 1000 & 920.6 & 915.8 \\
\hline Shear viscosity (Pa s) & 0.000891 & 0.00663 & 0.054 & 0.092 \\
\hline Attenuation coefficient $\left(\mathrm{Np} \mathrm{m}^{-1} \mathrm{MHz}^{-\mathrm{p}}\right)$ & 0.023 & 0.145 & 1.15 & 1.625 \\
\hline Attenuation exponent $\mathrm{p}$ & 2 & 2 & 1.77 & 2 \\
\hline
\end{tabular}

viscosity of the fluid medium increases, the inflection of the real part of the normalised effective viscosity moves to higher frequency [but corresponds to the same value of $\left.\operatorname{Re}\left(k_{s} a\right)\right]$.

\section{CONCLUSION}

An effective medium core-shell model has been utilised, taking mode conversions into account, to study the case of spherical solid particles in a viscous liquid host, deriving the effective bulk modulus and mass density for the system. Approximate explicit formulas for both effective properties have been derived under the condition of large compressional wavelengths in the particles, the host, and the effective medium, with respect to the particle size. No assumption was made on the shear wavelength in the host, allowing the study of the transition of the effective mass density from its volume averaged value at large shear wavelength to Ament's formula for an inviscid fluid at small ones. The effective density is both frequency and concentration dependent within the long compressional wavelength region, given by Eqs. (4), (8), and (11), representing, respectively, the general dependence, the leading order dependence in compressional wavenumber, and a low frequency expansion in the shear wavenumber.

Numerical calculations for silica in water systems show that the frequency and concentration dependence of the effective density in a viscous liquid host medium is complex and complicated. It exhibits a resonance behaviour that occurs at increasing values of $\operatorname{Re}\left(k_{s} a\right)$ as the concentration increases. This is consistent with previous observations of a similar behavior of the coherent wave effective properties made during the comparison of a multi-mode multiple scattering model with experimental data. ${ }^{41-43}$

The analytical results presented account for viscous effects in modelling the dynamics of nanoparticle systems. We chose to follow the same procedure as the authors of Ref. 17, working directly on the boundary conditions at the shell outer surface with zero amplitude scattered waves. In Ref. 17, the authors were able to derive not only the effective mass density and bulk modulus, but also the shear modulus, which allows the reconstruction of the effective wavenumbers of the coherent waves. In our, apparently more complicated, case, work is still in progress to obtain the wavenumbers, and it may be that a criterion for large shear wavelength may be required in order to do so. We hope that way, not only to recover the same attenuation of the coherent compressional wave as from the MST model ${ }^{39}$ for small concentrations, as validated by experiments, ${ }^{42}$ but also to be able to predict that attenuation for higher concentrations than the MST-based model can account for.

\section{ACKNOWLEDGMENTS}

The authors gratefully acknowledge funding for M.M.A. from the University of Le Havre.

\section{APPENDIX}

\section{Monopole and dipole scattering coefficients of a single sphere}

The propagating displacement field $\mathbf{u}$ associated with a mode of order $n$ in the host is expressed using Debye potentials $^{60}$ either as $\mathbf{u}=\nabla \varphi$ (with a different sign compared to Ref. 40) for a compressional wave or as $\mathbf{u}=\nabla \wedge \nabla \wedge\left(\varphi r \mathbf{e}_{r}\right)$ for a shear wave, with $\varphi=j_{n}\left(k_{\alpha} r\right) P_{n}(\cos \theta) \exp (-i \omega t)$ for the incident mode, and $\varphi=T_{n}^{\alpha \beta} h_{n}\left(k_{\beta} r\right) P_{n}(\cos \theta) \exp (-i \omega t)$ for the scattered wave in the spherical coordinates system $(r, \theta, \phi)$ centred at the sphere centre $\left(h_{n}\right.$ denoting the spherical Hankel function $h_{n}^{(1)}$ of the first kind). The scattering coefficients are obtained by solving the boundary equations (radial and tangential displacement and stress) at the particle surface.

When the compressional wavelength in both host and sphere is much larger than the particle radius, the dominant term of the only non-zero monopole scattering coefficient is, after Refs. 49 and 61 for an elastic solid host and Ref. 62 for a viscous fluid host,

$$
T_{0}^{c c}=i \frac{\left(B-B^{\prime}\right)\left(k_{c} a\right)^{3}}{4 \mu+3 B^{\prime}},
$$

where $B=\lambda+\frac{2}{3} \mu$ and $B^{\prime}=\lambda^{\prime}+\frac{2}{3} \mu^{\prime}$ are the bulk modulus of the matrix and the particle, respectively.

The dominant terms of the dipole scattering coefficients of an elastic sphere in a viscous fluid under the large compressional wavelengths condition are given in Ref. 41 and can be written as (with different signs for both $T_{1}^{c s}$ and $T_{1}^{s c}$ due to a different convention in the definition of the potentials associated with compressional waves)

$$
\begin{aligned}
& T_{1}^{c c}=\frac{i\left(k_{c} a\right)^{3} \beta_{h a}}{3 Y}(\hat{\rho}-1)=\left(k_{c} b\right)^{3} \tilde{T}_{1}^{c c}, \\
& T_{1}^{c s}=-\frac{\left(k_{c} a\right)}{Y}(\hat{\rho}-1)=\left(k_{c} b\right) \tilde{T}_{1}^{c s}, \\
& T_{1}^{s c}=-2 \frac{\left(k_{c} a\right)^{2}}{\left(k_{S} a\right) Y}(\hat{\rho}-1)=\left(k_{c} b\right)^{2} \tilde{T}_{1}^{s c},
\end{aligned}
$$




$$
T_{1}^{s s}=-\frac{\left[3 \beta_{j a}+2(\hat{\rho}-1) \gamma_{j a}\right]}{Y}=\tilde{T}_{1}^{s s},
$$

with

$$
\begin{aligned}
& Y=3 \beta_{h a}+2(\hat{\rho}-1) \gamma_{h a}, \\
& \beta_{h a}=k_{s} a h_{1}^{\prime}\left(k_{S} a\right)-h_{1}\left(k_{s} a\right) \quad \beta_{j a}=k_{s} a j_{1}^{\prime}\left(k_{s} a\right)-j_{1}\left(k_{s} a\right), \\
& \gamma_{h a}=k_{s} a h_{1}^{\prime}\left(k_{s} a\right)+2 h_{1}\left(k_{s} a\right) \quad \gamma_{j a}=k_{s} a j_{1}^{\prime}\left(k_{s} a\right)+2 j_{1}\left(k_{s} a\right),
\end{aligned}
$$

and

$$
\hat{\rho}=\frac{\rho^{\prime}}{\rho} .
$$

Equation (A4) has been obtained from Eq. (24) in Ref. 40 after using the value of the Wronskian between the spherical Bessel functions of the first and second kind, ${ }^{60} W\left[j_{n}(z), y_{n}(z)\right]=z^{-2}$.

Considering a viscous liquid host, with large $\left|k_{s} a\right|$, but still within the long compressional wavelength limit, the dimensionless shear wavenumber in the viscous host fluid, $k_{s} a=(1+i) \sqrt{\left(\omega \rho / 2 \eta_{S}\right)} a$, is written in the form

$$
k_{s} a=(1+i) x
$$

with $x \gg 1$. Using the asymptotic expansions of the spherical Bessel and Hankel functions, in Eqs. (A2)-(A7) for large $x$, leads to

$$
\begin{aligned}
& T_{1}^{c c} \simeq \frac{i}{3}\left(k_{c} a\right)^{3} \frac{\hat{\rho}-1}{1+2 \hat{\rho}}, \\
& T_{1}^{c s} \simeq-i e^{-i k_{s} a}\left(k_{c} a\right) \frac{\hat{\rho}-1}{1+2 \hat{\rho}}, \\
& T_{1}^{s c} \simeq-2 i \frac{e^{-i k_{s} a}}{k_{s} a}\left(k_{c} a\right)^{2} \frac{\hat{\rho}-1}{1+2 \hat{\rho}}, \\
& T_{1}^{s s} \simeq-i \sin \left(k_{s} a\right) e^{-i k_{s} a} .
\end{aligned}
$$

Equation (A10) is identical to that obtained for a single sphere in an inviscid host liquid which does not support shear waves.

The second equality in Eqs. (A2)-(A5), introducing the tilde scattering coefficients, and exhibiting the dependence of the scattering coefficients on $k_{c} b$ are valid whatever the inner structure of the spherical particles, and based only on the assumption that $\left|k_{c} b\right|$ is small.

\section{Determination of effective bulk modulus and mass density}

The effective properties are obtained by applying the same boundary equations as in the single sphere case to the outer boundary, $r=b$ of the core-shell system of the effective medium model. In the shell, the wave potentials are written (for an incident compressional wave) as

$$
\begin{aligned}
\Phi_{c, \text { shell }}= & \Phi_{0} \sum_{n=0}^{\infty} i^{n}(2 n+1) P_{n}(\cos \theta) \\
& \times\left\{A_{n}^{c c} j_{n}\left(k_{c} r\right)+\left(A_{n}^{c c} T_{n}^{c c}+A_{n}^{c s} T_{n}^{s c}\right) h_{n}\left(k_{c} r\right)\right\},
\end{aligned}
$$

$$
\begin{aligned}
\psi_{s, \text { shell }}= & \Phi_{0} \sum_{n=1}^{\infty} i^{n}(2 n+1) P_{n}(\cos \theta) \\
& \times\left\{A_{n}^{c s} j_{n}\left(k_{s} r\right)+\left(A_{n}^{c c} T_{n}^{c s}+A_{n}^{c s} T_{n}^{s s}\right) h_{n}\left(k_{s} r\right)\right\} .
\end{aligned}
$$

Scattered waves in the embedding medium are set to have zero amplitude due to the use of the self-consistency condition. The monopole boundary equations used to obtain the effective bulk modulus (radial displacement and radial stress component) are therefore

$$
\begin{aligned}
& K_{c} b j_{0}^{\prime}\left(K_{c} b\right)=A_{0}^{c c} k_{c} b j_{0}^{\prime}\left(k_{c} b\right)+T_{0}^{c c} A_{0}^{c c} k_{c} b h_{0}^{\prime}\left(k_{c} b\right) \\
& -\left(\lambda_{e f f}+2 \mu_{e f f}\right)\left(K_{c} b\right)^{2} j_{0}\left(K_{c} b\right)-4 \mu_{e f f} K_{c} b j_{0}^{\prime}\left(K_{c} b\right) \\
& =A_{0}^{c c}\left[-(\lambda+2 \mu)\left(k_{c} b\right)^{2} j_{0}\left(k_{c} b\right)-4 \mu k_{c} b j_{0}^{\prime}\left(k_{c} b\right)\right] \\
& \quad+T_{0}^{c c} A_{0}^{c c}\left[-(\lambda+2 \mu)\left(k_{c} b\right)^{2} h_{0}\left(k_{c} b\right)-4 \mu k_{c} b h_{0}^{\prime}\left(k_{c} b\right)\right] .
\end{aligned}
$$

Retaining only the dominant terms of the Bessel functions in the large compressional wavelength assumption leads to Eq. (1).

For the dipole mode, the four boundary conditions at $r=b$ that are the starting point to derive the effective mass density are (in the order radial displacement, tangential displacement, radial stress, tangential stress)

$$
\begin{aligned}
& K_{c} b j_{1}^{\prime}\left(K_{c} b\right)= A_{1}^{c c}\left[k_{c} b j_{1}^{\prime}\left(k_{c} b\right)+T_{1}^{c c} k_{c} b h_{1}^{\prime}\left(k_{c} b\right)\right. \\
&\left.+2 T_{1}^{c s} h_{1}\left(k_{s} b\right)\right]+A_{1}^{c s}\left[2 j_{1}\left(k_{s} b\right)\right. \\
&\left.+T_{1}^{s c} k_{c} b h_{1}^{\prime}\left(k_{c} b\right)+2 T_{1}^{s s} h_{1}\left(k_{s} b\right)\right], \quad(\mathrm{A} 18) \\
& j_{1}\left(K_{c} b\right)=A_{1}^{c c}\left\{\begin{array}{c}
j_{1}\left(k_{c} b\right)+T_{1}^{c c} h_{1}\left(k_{c} b\right) \\
+T_{1}^{c s}\left[k_{s} b h_{1}^{\prime}\left(k_{s} b\right)+h_{1}\left(k_{s} b\right)\right]
\end{array}\right\} \\
&+A_{1}^{c s}\left\{\begin{array}{c}
{\left[k_{s} b j_{1}^{\prime}\left(k_{s} b\right)+j_{1}\left(k_{s} b\right)\right]+T_{1}^{s c} h_{1}\left(k_{c} b\right)} \\
+T_{1}^{s s}\left[k_{s} b h_{1}^{\prime}\left(k_{s} b\right)+h_{1}\left(k_{s} b\right)\right]
\end{array}\right\},
\end{aligned}
$$

$$
\begin{aligned}
& \frac{\rho_{\text {eff }}}{\left(K_{s} b\right)^{2}}\left\{\begin{array}{c}
\left.\left[\left(K_{s} b\right)^{2}-4\right] j_{1}\left(K_{c} b\right)\right\} \\
+4 K_{c} b j_{1}^{\prime}\left(K_{c} b\right)
\end{array}\right\} \\
& =A_{1}^{c c} \frac{\rho}{\left(k_{s} b\right)^{2}}\left\{\left[\left(k_{s} b\right)^{2}-4\right] j_{1}\left(k_{c} b\right)+4 k_{c} b j_{1}^{\prime}\left(k_{c} b\right)\right\} \\
& +A_{1}^{c c} T_{1}^{c c} \frac{\rho}{\left(k_{s} b\right)^{2}}\left\{\left[\left(k_{s} b\right)^{2}-4\right] h_{1}\left(k_{c} b\right)+4 k_{c} b h_{1}^{\prime}\left(k_{c} b\right)\right\} \\
& +4 A_{1}^{c c} T_{1}^{c s} \frac{\rho}{\left(k_{s} b\right)^{2}}\left[h_{1}\left(k_{s} b\right)-k_{s} b h_{1}^{\prime}\left(k_{s} b\right)\right] \\
& +4 A_{1}^{c s} \frac{\rho}{\left(k_{s} b\right)^{2}}\left[j_{1}\left(k_{s} b\right)-k_{s} b j_{1}^{\prime}\left(k_{s} b\right)\right] \\
& +A_{1}^{c s} T_{1}^{s c} \frac{\rho}{\left(k_{s} b\right)^{2}}\left\{\left[\left(k_{s} b\right)^{2}-4\right] h_{1}\left(k_{c} b\right)+4 k_{c} b h_{1}^{\prime}\left(k_{c} b\right)\right\} \\
& +4 A_{1}^{c s} T_{1}^{s s} \frac{\rho}{\left(k_{s} b\right)^{2}}\left[h_{1}\left(k_{s} b\right)-k_{s} b h_{1}^{\prime}\left(k_{s} b\right)\right],
\end{aligned}
$$




$$
\begin{aligned}
& 2 \frac{\rho_{\text {eff }}}{\left(K_{s} b\right)^{2}}\left[j_{1}\left(K_{c} b\right)-\left(K_{c} b\right) j_{1}^{\prime}\left(K_{c} b\right)\right] \\
& =2 A_{1}^{c c} \frac{\rho}{\left(k_{s} b\right)^{2}}\left[j_{1}\left(k_{c} b\right)-k_{c} b j_{1}^{\prime}\left(k_{c} b\right)\right] \\
& \quad+2 A_{1}^{c c} T_{1}^{c c} \frac{\rho}{\left(k_{s} b\right)^{2}}\left\{h_{1}\left(k_{c} b\right)-k_{c} b h_{1}^{\prime}\left(k_{c} b\right)\right\} \\
& \quad+A_{1}^{c c} T_{1}^{c s} \frac{\rho}{\left(k_{s} b\right)^{2}}\left\{2 k_{s} b h_{1}^{\prime}\left(k_{s} b\right)+\left[\left(k_{s} b\right)^{2}-2\right] h_{1}\left(k_{s} b\right)\right\} \\
& \quad+A_{1}^{c s} \frac{\rho}{\left(k_{s} b\right)^{2}}\left\{2 k_{s} b j_{1}^{\prime}\left(k_{s} b\right)+\left[\left(k_{s} b\right)^{2}-2\right] j_{1}\left(k_{s} b\right)\right\} \\
& \quad+2 A_{1}^{c s} T_{1}^{s c} \frac{\rho}{\left(k_{s} b\right)^{2}}\left[h_{1}\left(k_{c} b\right)-k_{c} b h_{1}^{\prime}\left(k_{c} b\right)\right] \\
& \quad+A_{1}^{c s} T_{1}^{s s} \frac{\rho}{\left(k_{s} b\right)^{2}}\left\{2 k_{s} b h_{1}^{\prime}+\left[\left(k_{s} b\right)^{2}-2\right] h_{1}\left(k_{s} b\right)\right\} .
\end{aligned}
$$

Equations (4) and (5) have been obtained by multiplying Eq. (A21) by 2, adding it to Eq. (A20), and dividing by Eq. (A19). The ratio of the scattering coefficients, $D=A_{1}^{c c} / A_{1}^{c s}$, as given in Eq. (7), is obtained from the subtraction of Eq. (A19) from Eq. (A18) and the neglect of terms of the order $\left(K_{c} b\right)^{3}$ on the left hand side, under the condition that $A_{1}^{c c}$ is of order less than or equal to 1 [i.e., $O\left(k_{c} b\right)^{1}$ ] and $A_{1}^{c s}$ is of order less than or equal to 2 [i.e., $O\left(k_{c} b\right)^{2}$ ]. In order to validate those assumptions, MAPLE(Maplesoft) software has been used to carry out analytical expansions under the large compressional wavelength assumptions, $\left|k_{c} b\right| \ll 1,\left|K_{c} b\right| \ll 1$. First, Eqs. (A18) and (A19) have been solved to obtain $A_{1}^{c c}$ and $A_{1}^{c s}$. Using then the expansions of the Bessel and Hankel functions associated with compressional waves in series of powers of their argument, and taking into account the dependency of the scattering coefficients defined in Eqs. (A2)-(A7) on $k_{c} b$, their respective leading order terms are found to be, respectively, $O\left(k_{c} b\right)^{0}$ and $O\left(k_{c} b\right)^{1}$. The dominant term of $D$ is an approximation of Eq. (7) and can be obtained from those leading order terms as

$$
D \simeq\left(\frac{1}{k_{c} b}\right) \frac{\tilde{T}_{1}^{s s} \beta_{h b}+\beta_{j b}-3 i \tilde{T}_{1}^{s c}}{\tilde{T}_{1}^{c s} \beta_{h b}-3 i \tilde{T}_{1}^{c c}} .
$$

Introduction of Eq. (A22) into Eq. (4) then provides the leading order in $k_{c} b$ of the effective mass density as in Eq. (8).

The expansion of the effective mass density in powers of $k_{s} b$, Eq. (11), has been obtained after using expansions of all the Bessel and Hankel functions related to shear waves, including those in the "tilde" scattering coefficients, up to relatively large orders, because a large number of terms cancel each other. Since $\left(k_{s} b\right)^{2}$ is purely imaginary for a viscous fluid, it is necessary to retain the first two leading order terms in the effective density in order to obtain both the real and imaginary part of the frequency-dependent effective density.

In order to obtain the limit of effective density for an inviscid medium, $\left|k_{s} a\right|,\left|k_{s} b\right|$ are taken to be large, using the asymptotic expansions of the Bessel and Hankel functions in Eq. (8), along with the scattering coefficients for large $\left|k_{s} b\right|$ [Eqs. (A10)-(A13)]. The numerator of the fraction in Eq. (8) thus behaves as

$$
i \frac{c}{3}\left(k_{c} a\right)^{3} e^{-i k_{s} a} \sin \left(k_{s} a-k_{c} b\right) \frac{\rho-1}{1+2 \rho},
$$

and the denominator as

$$
\left(k_{c} a\right)^{3} e^{-i k_{s} a} \sin \left(k_{s} a-k_{c} b\right) \frac{-1-2 c+2(c-1) \hat{\rho}}{1+2 \hat{\rho}},
$$

so that Eq. (8) becomes Eq. (15) in the limit of large $\left|k_{s} a\right|,\left|k_{s} b\right|$.

For an inviscid liquid host which does not support shear

\begin{tabular}{|c|c|}
\hline & \\
\hline$A_{n}^{c c}, A_{n}^{c s}$ & $\begin{array}{l}n \text {th mode refracted amplitude of com- } \\
\text { pressional and shear waves, respec- } \\
\text { tively, generated from an incident } \\
\text { effective compressional mode }\end{array}$ \\
\hline$c$ & Volume concentration \\
\hline$v$ & Sound speed in the host \\
\hline$\alpha$ & $\begin{array}{l}\text { Attenuation of the compressional } \\
\text { waves in the host }\end{array}$ \\
\hline$\eta_{s}$ & Shear viscosity of the host \\
\hline$\lambda, \mu$ & Lamé parameters \\
\hline$K_{c}, K_{s}$ & $\begin{array}{l}\text { Effective wavenumbers of compres- } \\
\text { sional and shear wave modes }\end{array}$ \\
\hline$\omega$ & Angular frequency \\
\hline$k_{c}=\frac{\omega}{v}+i \alpha$ & $\begin{array}{l}\text { Compressional wavenumber in the vis- } \\
\text { cous fluid host medium }\end{array}$ \\
\hline$(+i)$ & $\begin{array}{l}\text { Shear wavenumber in the viscous fluid } \\
\text { host medium }\end{array}$ \\
\hline
\end{tabular}
waves (rather than the limit of a viscous liquid host at large $\left.\left|k_{s} a\right|\right)$, the boundary equations at $r=b$ are greatly simplified, resulting in

$$
\begin{gathered}
K_{c} b j_{n}^{\prime}\left(K_{c} b\right)=k_{c} b A_{n}^{c c}\left[j_{n}^{\prime}\left(k_{c} b\right)+T_{n}^{c c} h_{n}^{\prime}\left(k_{c} b\right)\right], \\
\frac{\rho_{e f f}}{\rho} j_{n}\left(K_{c} b\right)=A_{n}^{c c}\left[j_{n}\left(k_{c} b\right)+T_{n}^{c c} h_{n}\left(k_{c} b\right)\right] .
\end{gathered}
$$

This leads to Eq. (3) for the monopole mode $n=0$ and, for the dipole mode $n=1$, to Eq. (15), which is the limit of large $\left|k_{s} b\right|$ in a viscous liquid host. ${ }^{63}$

\section{NOMENCLATURE}

${ }^{1}$ R. Hill, "A self-consistent mechanics of composite materials," J. Mech. Phys. Solids 13, 213-222 (1965).

${ }^{2}$ B. Budiansky, "On the elastic moduli of some heterogeneous materials," J. Mech. Phys. Solids 13, 223-227 (1965).

${ }^{3}$ R. M. Christensen and K. H. Lo, "Solutions for effective shear properties in three phase sphere and cylinder models," J. Mech. Phys. Solids 27, 315-330 (1979).

${ }^{4}$ E. H. Kerner, "The elastic and thermo-elastic properties of composite media," Proc. Phys. Soc. Sect. B 69, 808-813 (1956).

${ }^{5}$ R. M. Christensen, "A critical evaluation for a class of micro-mechanics models," in Inelastic Deformation of Composite Materials, edited by G. J. Dvorak, International Union of Theoretical and Applied Mechanics (Springer, New York, 1991), pp. 275-282.

${ }^{6} \mathrm{Y}$. Benveniste, "Effective thermal conductivity of composites with a thermal contact resistance between the constituents: Nondilute case," J. Appl. Phys. 61, 2840-2843 (1987). 
${ }^{7}$ H. A. Luo and G. J. Weng, "On Eshelby's inclusion problem in a threephase spherically concentric solid, and a modification of Mori-Tanaka's method," Mech. Mater. 6, 347-361 (1987).

${ }^{8}$ G. Siboni and Y. Benveniste, "A micromechanics model for the effective thermomechanical behaviour of multiphase composite media," Mech. Mater. 11, 107-122 (1991).

${ }^{9}$ Y. Huang, K. X. Hu, X. Wei, and A. Chandra, "A generalized selfconsistent mechanics method for composite materials with multiphase inclusions," J. Mech. Phys. Solids 42, 491-504 (1994).

${ }^{10} \mathrm{~S}$. Biwa, N. Ito, and N. Ohno, "Elastic properties of rubber particles in toughened PMMA: Ultrasonic and micromechanical evaluation," Mech. Mater. 33, 717-728 (2001).

${ }^{11}$ S. K. Kanaun, V. M. Levin, and F. J. Sabina, "Propagation of elastic waves in composites with random set of spherical inclusions (effective medium approach)," Wave Motion 40, 69-88 (2004).

${ }^{12}$ F. J. Sabina and J. R. Willis, "A simple self-consistent analysis of wave propagation in particulate composites," Wave Motion 10, 127-142 (1988).

${ }^{13}$ J. G. Berryman, "Theory of elastic properties of composite materials," Appl. Phys. Lett. 35, 856-858 (1979).

${ }^{14}$ J. G. Berryman, "Long-wavelength propagation in composite elastic media. II. Ellipsoidal inclusions,” J. Acoust. Soc. Am. 68, 1820-1831 (1980).

${ }^{15}$ G. T. Kuster and M. N. Toksöz, "Velocity and attenuation of seismic waves in two-phase media: Part I. Theoretical formulations," Geophysics 39, 587-606 (1974).

${ }^{16} \mathrm{C}$. Jin, "On the estimation of dynamic mass density of random composites," J. Acoust. Soc. Am. 132, 615-620 (2012).

${ }^{17}$ J. Mei, Z. Liu, W. Wen, and P. Sheng, "Effective dynamic mass density of composites," Phys. Rev. B 76, 134205 (2007).

${ }^{18}$ G. C. Gaunaurd and H. Überall, "Resonance effects and the ultrasonic effective properties of particulate composites," J. Acoust. Soc. Am. 74, 305-313 (1983).

${ }^{19} \mathrm{~F}$. H. Kerr, "An effective medium approach to the study of plane wave propagation in an elastic matrix with spherical elastic inclusions," Int. J. Eng. Sci. 30, 187-198 (1992).

${ }^{20} \mathrm{~J}$. Kim, J. Ih, and B. Lee, "Dispersion of elastic waves in random particulate composites," J. Acoust. Soc. Am. 97, 1380-1388 (1995).

${ }^{21}$ Y. Hemar, N. Herrmann, P. Lemarechal, R. Hocquart, and F. Lequeux, "Effective medium model for ultrasonic attenuation due to the thermoelastic effect in concentrated emulsions," J. Phys. II France 7, 637-647 (1997).

${ }^{22}$ D. J. McClements, Y. Hemar, and N. Herrmann, "Incorporation of thermal overlap effects into multiple scattering theory," J. Acoust. Soc. Am. 105, 915-918 (1999).

${ }^{23}$ M. L. Cowan, K. Beaty, J. H. Page, Z. Liu, and P. Sheng, "Group velocity of acoustic waves in strongly scattering media: Dependence on the volume fraction of scatterers," Phys. Rev. E 58, 6626-6636 (1998).

${ }^{24}$ A. K. Hipp, G. Storti, and I. Morbidelli, "Acoustic characterization of concentrated suspensions and emulsions. 1. Model analysis," Langmuir 18, 391-404 (2002).

${ }^{25}$ L. L. Foldy, "The multiple scattering of waves," Phys. Rev. 67, 107-119 (1945).

${ }^{26}$ M. Lax, "Multiple scattering of waves. II. The effective field in dense systems," Phys. Rev. 85, 621-629 (1952).

${ }^{27}$ P. C. Waterman and R. Truell, "Multiple scattering of waves," J. Math. Phys. 2, 512-537 (1961).

${ }^{28}$ J. G. Fikioris and P. C. Waterman, "Multiple scattering of waves. II. 'Hole corrections' in the scalar case," J. Math. Phys. 5, 1413-1420 (1964).

${ }^{29} \mathrm{P}$. Lloyd and M. V. Berry, "Wave propagation through an assembly of spheres. IV. Relations between different multiple scattering theories," Proc. Phys. Soc. London 91, 678-688 (1967).

${ }^{30}$ V. K. Varadan, Y. Ma, and V. V. Varadan, "A multiple scattering theory for elastic wave propagation in discrete random media," J. Acoust. Soc. Am. 77, 375-385 (1985).

${ }^{31}$ V. K. Varadan, V. V. Varadan, and Y. Ma, "Multiple scattering of elastic waves by cylinders of arbitrary cross section II. Pair-correlated cylinders," J. Acoust. Soc. Am. 78, 1874-1878 (1985).

${ }^{32}$ C. M. Linton and P. A. Martin, "Multiple scattering by random configurations of circular cylinders: Second-order corrections for the effective wavenumber," J. Acoust. Soc. Am. 117, 3413-3423 (2005).
${ }^{33}$ C. M. Linton and P. A. Martin, "Multiple scattering by multiple spheres: A new proof of the Lloyd- Berry formula for the effective wavenumber," SIAM J. Appl. Math. 66, 1649-1668 (2006).

${ }^{34}$ W. J. Parnell, I. D. Abrahams, and P. R. Brazier-Smith, "Effective properties of a composite half-space: Exploring the relationship between homogenization and multiple-scattering theories," Quart. J. Mech. Appl. Math. 63, 145-175 (2010).

${ }^{35}$ M. Caleap, B. W. Drinkwater, and P. D. Wilcox, "Coherent acoustic wave propagation in media with pair-correlated spheres," J. Acoust. Soc. Am. 131, 2036-2047 (2012).

${ }^{36}$ P. A. Martin, A. Maurel, and W. J. Parnell, "Estimating the dynamic effective mass density of random composites," J. Acoust. Soc. Am. 128, 571-577 (2010).

${ }^{37}$ C. Aristegui and Y. C. Angel, "Effective mass density and stiffness derived from P-wave multiple scattering," Wave Motion 44, 153-164 (2007).

${ }^{38}$ J. M. Conoir and A. N. Norris, "Effective wavenumbers and reflection coefficients for an elastic medium containing random configurations of cylindrical scatterers," Wave Motion 47, 183-197 (2010).

${ }^{39}$ F. Luppe, J. M. Conoir, and A. N. Norris, "Effective wave numbers for thermo-viscoelastic media containing random configurations of spherical scatterers," J. Acoust. Soc. Am. 131, 1113-1120 (2012).

${ }^{40}$ F. Luppé, T. Valier-Brasier, J. M. Conoir, and P. Pareige, "Coherent wave propagation in viscoelastic media with mode conversions and paircorrelated scatterers," Wave Motion 72, 244-259 (2017).

${ }^{41}$ V. J. Pinfield and D. M. Forrester, "Multiple scattering in random dispersions of spherical scatterers: Effects of shear-acoustic interactions," J. Acoust. Soc. Am. 141, 649-660 (2017).

${ }^{42}$ D. M. Forrester, J. Huang, V. J. Pinfield, and F. Luppé, "Experimental verification of nanofluid shear-wave re- conversion in ultrasonic fields," Nanoscale 8, 5497-5506 (2016).

${ }^{43}$ D. M. Forrester, J. Huang, and V. J. Pinfield, "Characterisation of colloidal dispersions using ultrasound spectroscopy and multiple-scattering theory inclusive of shear-wave effects," Chem. Eng. Res. Des. 114, 69-78 (2016).

${ }^{44}$ R. E. Challis and V. J. Pinfield, "Ultrasonic wave propagation in concentrated slurries-The modelling problem," Ultrasonics 54, 1737-1744 (2014).

${ }^{45}$ J. Dubois, C. Aristegui, O. Poncelet, and A. L. Shuvalov, "Coherent acoustic response of a screen containing a random distribution of scatterers: Comparison between different approaches," J. Phys. Conf. Ser. 269, 012004 (2011).

${ }^{46}$ R.-B. Yang and A. K. Mal, "Multiple scattering of elastic waves in a fiber-reinforced composite," J. Mech. Phys. Solids 42, 1945-1968 (1994).

${ }^{47}$ R.-B. Yang, "A dynamic generalized self-consistent model for wave propagation in particulate composites," J. Appl. Mech. 70, 575-582 (2003).

${ }^{48}$ J.-Y. Kim, "On the generalized self-consistent model for elastic wave propagation in composite materials," Int. J. Solids Struct. 41, 4349-4360 (2004).

${ }^{49}$ R. L. Kligman, W. M. Madigosky, and J. R. Barlow, "Effective dynamic properties of composite viscoelastic materials," J. Acoust. Soc. Am. 70, 1437-1444 (1981).

${ }^{50} \mathrm{X}$. Jing, P. Sheng, and M. Zhou, "Acoustic and electromagnetic quasimodes in dispersed random media," Phys. Rev. A 46, 6513-6534 (1992).

${ }^{51}$ Y. Wu, J. Li, Z. Q. Zhang, and C. T. Chan, "Effective medium theory for magnetodielectric composites: Beyond the long-wavelength limit," Phys. Rev. B 74, 085111 (2006)

${ }^{52}$ A. B. Wood, A Textbook of Sound (Bell, London, 1941).

${ }^{53}$ J. R. Allegra and S. A. Hawley, "Attenuation of sound in suspensions and emulsions: Theory and experiments," J. Acoust. Soc. Am. 51, 1545-1564 (1972).

${ }^{54}$ W. S. Ament, "Sound propagation in gross mixtures," J. Acoust. Soc. Am. 25, 638-641 (1953).

${ }^{55}$ M. Caleap, B. W. Drinkwater, and P. D. Wilcox, "Effective dynamic constitutive parameters of acoustic metamaterials with random microstruture," New J. Phys. 14, 033014 (2012).

${ }^{56} \mathrm{D}$. Torrent and J. Sánchez-Dehesa, "Effective parameters of clusters of cylinders embedded in a nonviscous fluid or gas," Phys. Rev. B 74, 224305 (2006).

${ }^{57}$ J. Mei, Z. Liu, W. Wen, and P. Sheng, "Effective mass density of fluidsolid composites," Phys. Rev. Lett. 96, 024301 (2006). 
${ }^{58}$ D. J. McClements and M. J. W. Povey "Scattering of ultrasound by emulsions," J. Phys. D. Appl. Phys. 22, 38-47 (1989).

${ }^{59}$ R. E. Challis, M. J. W. Povey, M. L. Mather, and A. K. Holmes, "Ultrasound techniques for characterizing colloidal dispersions," Rep. Prog. Phys. 68, 1541-1637 (2005).

${ }^{60}$ D. Brill, G. Gaunaurd, and H. Überall, "Resonance theory of elastic shearwave scattering from spherical fluid obstacles in solids," J. Acoust. Soc. Am. 67, 414-424 (1980).
${ }^{61}$ C. F. Ying and R. Truell, "Scattering of a plane longitudinal wave by a spherical obstacle in an isotropically elastic solid," J. Appl. Phys. 27, 1086-1097 (1956).

${ }^{62}$ P. S. Epstein and R. R. Carhart, "The absorption of sound in suspensions and emulsions. I. Water fog in air," J. Acoust. Soc. Am. 25, 553-565 (1953).

${ }^{63}$ J. N. Coupland and D. J. McClements, "Physical properties of liquid edible oils,” J. Am. Oil Chem. Soc. 74, 1559-1564 (1997). 